\title{
Photoconductive Multiplexing by ZnO:Zr:F Thin Solid Films
}

\author{
C. Torres-Torres, ${ }^{1}$ L. Castañeda, ${ }^{2}$ and R. Torres-Martínez ${ }^{3}$ \\ ${ }^{1}$ Sección de Estudios de Posgrado e Investigación, ESIME ZAC, Instituto Politécnico Nacional, 07738 México, DF, Mexico \\ ${ }^{2}$ Escuela Superior de Ingeniería Mecánica y Eléctrica Unidad Ticomán, Instituto Politécnico Nacional, 07340 México, DF, Mexico \\ ${ }^{3}$ Centro de Investigación en Ciencia Aplicada y Tecnología Avanzada Unidad Querétaro, Instituto Politécnico Nacional, \\ 76090 Santiago de Querétaro, QRO, Mexico \\ Correspondence should be addressed to C. Torres-Torres; crstorres@yahoo.com.mx
}

Received 30 May 2013; Accepted 1 August 2013

Academic Editor: Charles Sorrell

Copyright (C) 2013 C. Torres-Torres et al. This is an open access article distributed under the Creative Commons Attribution License, which permits unrestricted use, distribution, and reproduction in any medium, provided the original work is properly cited.

\begin{abstract}
Within this work, the nonlinear optical properties and electrical effects exhibited by zinc oxide thin films codoped with zirconium and fluorine are reported. The development of a simple photoconductive multiplexor system is proposed. The samples were prepared by the ultrasonic spraying technique (UST). Spectroscopic studies and a vectorial two-wave mixing method were carried out with a nanosecond Nd:YAG laser system at $532 \mathrm{~nm}$. Experimental results indicate that after the zirconium and fluorine doping, a strong third-order optical nonlinearity can be developed in the $\mathrm{ZnO}$ films. The nonlinear optical response seems to be dominantly originated by a two-photon absorption closely related to a photoconductive phenomenon.
\end{abstract}

\section{Introduction}

There has been a renewed interest in the improvement of new nonlinear optical (NLO) materials for developing novel applications in photonics and integrated optics. The demand of low-cost and high-performance optoelectronic systems has given rise to a progress related to more efficient transparent conductive oxide (TCO) thin films. Regarding the popular TCOs, last decade has seen zinc oxide [ $\mathrm{ZnO}$ ] to emerge as one of the most important materials for manufacturing transparent electrodes. Besides their high optical transparency, the $\mathrm{ZnO}$ thin films are highly stable in the hydrogen-plasma environment commonly used for the fabrication of silicon based $\mathrm{p}-\mathrm{i}-\mathrm{n}$ structures [1].

$\mathrm{ZnO}$ thin solid films have been deposited by a wide variety of techniques, and among those, the ultrasonic spray technique has been successfully employed for developing conductive and transparent $\mathrm{ZnO}$ thin films [2]. Keeping in mind the high optical transparency as one of the basic requirements for optoelectronic applications, the incorporation of distinct dopants has been carried out to reduce the resistivity of $\mathrm{ZnO}$ films [3]. Despite being similar to all the ultrasonic deposition processing routes, the ultrasonic spray technique involves numerous parameters and conditions to control the resulting physical properties of the $\mathrm{ZnO}$ thin films [4].

For electrode manufacturing, oriented $\mathrm{ZnO}$ films doped with adequate impurities are required to present a low resistivity; in this respect, stable $\mathrm{ZnO}$ thin films could be fabricated utilizing group-III elements as dopants [5], but frequently, an additional postdeposition annealing either in vacuum or under molecular hydrogen atmosphere has been performed to achieve a low resistivity [6]. Furthermore, incorporation of some other elements with higher valencies into the $\mathrm{ZnO}$ lattice has been contemplated as another chance in the quest for improving its optical and electrical characteristics [7]. It is worth noting to mention that it has been considered to establish the substitution of divalent $\mathrm{Zn}$ atoms from the lattice sites of $\mathrm{ZnO}$ by tetravalent zirconium [Zr] atoms to achieve changes in the optical and electrical features of $\mathrm{ZnO}: \mathrm{Zr}$ films [8].

On the other hand, anionic impurities like fluorine $[\mathrm{F}]$ also have been employed to reduce the resistivity with an additional enhancement of the nonlinear optical properties of the films, through the substitution of oxygen atoms of their lattice $[9,10]$.

Since the inclusion of both anionic and cationic impurities helps to reduce the resistivity of some metal oxide 


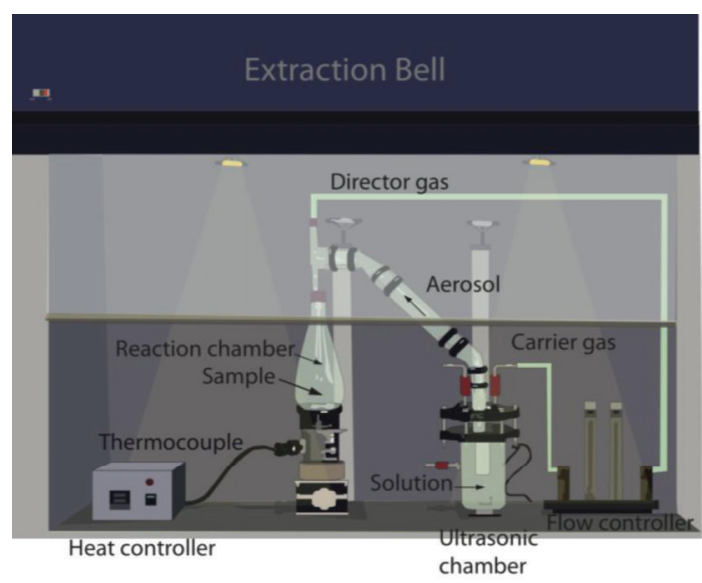

FIGURE 1: Schematic diagram of the experimental setup used for the deposition of the $\mathrm{ZnO}: \mathrm{Zr}$ :F thin solid films by the UST.

systems, in this work the incorporation of both $\mathrm{F}$ and $\mathrm{Zr}$ into the $\mathrm{ZnO}$ lattice seems to be attractive to study the resulting electrical and nonlinear optical properties. A UST was employed to prepare zinc oxide co-doped with zirconium and fluorine [ $\mathrm{ZnO}: \mathrm{Zr}: \mathrm{F}]$ thin solid films on glass substrates. The advantages observed in the electrical and optical phenomena allow us to present a photoconductive multiplexor system. A noticeable enhancement in the photoconductive and nonlinear optical responses of the films was observed. In order to explore their potentials in the development of optical devices, the experiments reported in this scientific research have been carried out as a fundamental study on the physical properties of $\mathrm{ZnO}: \mathrm{Zr}$ :F based samples deposited by an inexpensive UST.

\section{Experimental Details}

2.1. Fabrication of $\mathrm{ZnO}: \mathrm{Zr}: F$ Thin Solid Films. UST is a versatile method capable of producing nanostructured powder and thin film samples. The particle/grain size in these samples can be straightforwardly controlled by varying the concentration of the source solution and the atomization parameters. The deposition system used in this work includes a variable frequency piezoelectric transducer, which was set to $1.2 \mathrm{MHz}$ and operated at $120 \mathrm{~W}$ ultrasonic powers. In the UST system, a geyser is formed in the liquid surface by the action of a beam of ultrasonic vibrations which is directed to a liquid-gas interface. This geyser is followed by the generation of a spray formed by microscopic droplets. A carrier gas transports and directs the spray toward the surface of a hot substrate where the film formation is produced. The experimental setup used for the fabrication of $\mathrm{ZnO}: \mathrm{Zr}: \mathrm{F}$ films in this work is schematically presented in Figure 1 . The precursor solution used for the thin solid film deposition was prepared by dissolving zinc (II) pentanedionate $\left(\left[\mathrm{Zn}\left(\mathrm{C}_{5} \mathrm{H}_{7} \mathrm{O}_{2}\right) \cdot \mathrm{H}_{2} \mathrm{O}\right]\right.$, Alfa 98\%) and methanol ([ $\left.\mathrm{CH}_{3} \mathrm{OH}\right]$, Baker, 98\%) mixture $(12.5: 487.5, \mathrm{v} / \mathrm{v})$ under magnetic stirring to obtain a $0.2 \mathrm{M}$ concentration of $\mathrm{Zn}$ in it. After the complete dissolution of zinc precursor, measured amounts of zirconium (IV) acetylacetonate $\left(\left[\mathrm{Zr}\left(\mathrm{O}_{2} \mathrm{C}_{5} \mathrm{H}_{7}\right)_{4}\right]\right.$, Sigma-Aldrich, 98\%) and ammonium fluoride ( $\left[\mathrm{NH}_{4} \mathrm{~F}\right]$, Sigma-Aldrich, 48\%) were added to the mixture keeping $\mathrm{F}$ and atom $\mathrm{Zr} \%$ (nominal) 30.0 and $3.0 \%$ of $\mathrm{Zn}$ atoms. The $\mathrm{pH}$ of the final precursor solution was adjusted to 4 by adding excess acetic acid. While the concentrations of the dopants in the precursor solution were fixed taking into account that the addition of excess acetic acid or the final $\mathrm{pH}$ value of the precursor solution was to avoid an early precipitation of zinc hydroxide from it. The starting solution was prepared at room temperature in a dark and clean environment in order to avoid any photoinduced reaction. For ultrasonic spray, aliquots from the precursor solution were collected at different aging times. The thin films were deposited on glass substrates (alkali free borosilicate) with an area of $2.56 \times 2.56 \mathrm{~cm}^{2}$, and the substrates have a thickness of $0.5 \mathrm{~cm}$. Prior to film deposition, all the glass substrates were cleaned using the procedure as follows: (i) sonication for five minutes in Extranformaldehyde-free AP 33 (Merck, Millipore), followed by (ii) sonication for five minutes in trichloroethylene $\left(\left[\mathrm{C}_{2} \mathrm{HCl}_{3}\right]\right.$, Baker, 98\%) for degreasing the substrates, followed by (iii) sonication in methyl alcohol $\left(\left[\mathrm{CH}_{3} \mathrm{OH}\right]\right.$, Aldrich, 98\%), and (iiii) sonication in acetone $\left(\left[\mathrm{CH}_{3} \mathrm{COCH}_{3}\right]\right.$, Baker, $\left.98 \%\right)$, and finally, (v) the substrates are dried by a jet of pure and dry nitrogen ( $\left[\mathrm{N}_{2}\right]$, PRAXAIR, 99.997\%). Then the substrates were placed on a fused tin bath of the deposition system. The temperature of the substrates was measured by putting a chromel-alumel thermocouple placed below. The deposition system was covered with a stainless steel metal jacket during the pyrolysis process. Then, the substrate temperature serves to finish the chemical heterogeneous reaction that originates the $[\mathrm{ZnO}: \mathrm{Zr}: \mathrm{F}]$. The deposition time serves as a parameter to control the thickness of the samples. The substrate temperature $\left(T_{s}\right)$ varied from 450 to $500^{\circ} \mathrm{C}$ in $25^{\circ} \mathrm{C}$ steps, within an accuracy of $\pm 1^{\circ} \mathrm{C}$. This temperature range was chosen because in this case the samples present a low resistivity as $7.2 \times$ $10^{-2} \Omega \mathrm{cm}$. In that interval of the substrate temperature of the samples, on the part of element, Zinc there is no loss of mass, since its correspondent point of boil is $907^{\circ} \mathrm{C}$. Pure nitrogen $\left(\left[\mathrm{N}_{2}\right]\right.$, PRAXAIR, 99.997\%) was used both as the solution carrier and precursor directing gas, maintaining its flow rates at 3.5 and $0.5 \mathrm{~L} / \mathrm{min}$, respectively. The deposition time was set to $7.5 \mathrm{~min}$.

2.2. Characterization of ZnO:Zr:F Thin Solid Films. The crystallinity and microstructure of the deposited films were determined by X-ray diffraction (XRD) technique. XRD pattern of the sample in $\theta-2 \theta$ scan mode in the range of 30 to $80^{\circ}$ was recorded by utilizing the $\mathrm{Cu} \mathrm{K} \alpha_{1}(\lambda=0.15405 \mathrm{~nm})$ radiation. The morphology of the particles was analyzed in an XL FEG/SFEG/SIRION scanning electron microscope (FEI). The thickness of the films was measured using a KLA Tencor P15 profilometer with a resolution of $0.5 \mathrm{~nm}$. The measurement of optical transmittance of the studied samples was made by a UV-Visible 2401 PC Shimadzu spectrophotometer. The mechanism of the third-order nonlinearity in the samples was investigated by means of a vectorial two-wave mixing method [11]. A $532 \mathrm{~nm}$ wavelength and $1 \mathrm{~ns}$ pulse duration were provided by single shots from the second harmonic of an Nd-YAG laser source Continuum Model SL II-10 SN14419. 


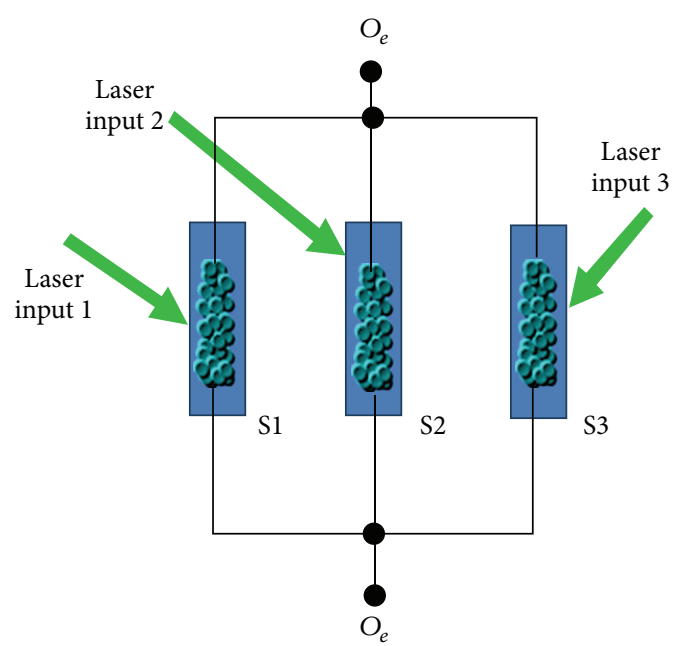

(a)

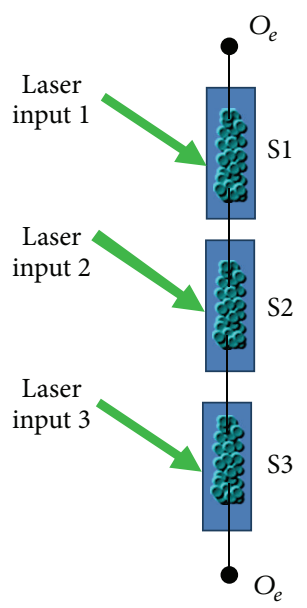

(b)

FIGURE 2: Schematic illustration of the setup for the photoconductive multiplexing system. (a) Parallel circuit. (b) Series circuit.

2.3. Photoconductive Multiplexor System. The operation principle underlying this function consists essentially in generating a change in the conductivity provided different $\mathrm{ZnO}: \mathrm{Zr}: \mathrm{F}$ samples separately exposed to optical beams. Figure 2 illustrates two different experimental setups: S1-3 represents the thin films and $O_{e}$ the electrical terminal points to conductivity measurement. The purpose of each configuration is to exhibit a different contribution of the electrical response of the photoconductive system when the optical beams are specifically turned on. In this way, there is a correspondent multiplexing effect collected by the photoconductive response of the system. The effect is derived by the participation of the optical signals that are present simultaneously in the circuit. The photoconductive responses for each of the samples were selectively excited by nonresonant irradiations of $532 \mathrm{~nm}$ wavelength and $1 \mathrm{~ns}$ pulse duration. The evaluation of the transmittance of this highirradiance single beam was carried out. The modification of the value of the electrical conductivity exhibited by the systems was separately measured during the propagation of the nanosecond pulses. The incident polarization of the beams was chosen to coincide with the path in measurement.

\section{Results and Discussions}

Figure 3 illustrates the XRD data associated to the samples.

From the XRD patterns, it is clear that all the samples were of polycrystalline nature. The XRD peak positions fit well to hexagonal wurtzite phase of $\mathrm{ZnO}$ (JCPDS no. 36-1451). No diffraction peak related to zirconium or any compound of zirconium or fluorine was detected. The samples revealed their preferential growth along the [002] direction, that is, (002) planes grown parallel to the substrate surface. Previous reports have indicated that it is supposed that $\mathrm{Zr}$ incorporation into the $\mathrm{Zn}$ sites is enhanced by the addition of $\mathrm{F}$ ions to the same starting solution [11]. However, there is no evidence of the presence of $\mathrm{F}$ and $\mathrm{Zr}$ dopants into the

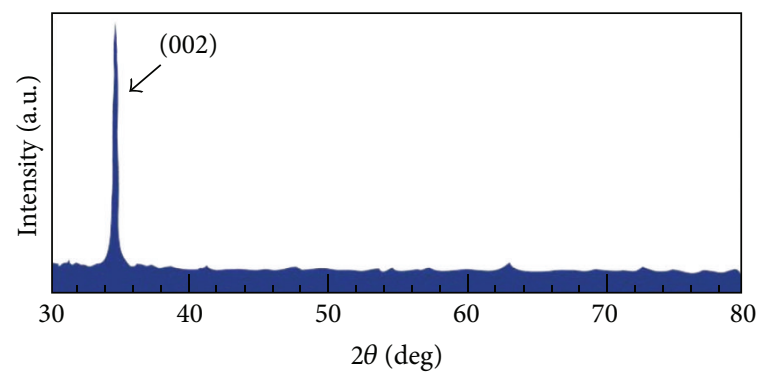

FIGURE 3: Representative XRD pattern of the $\mathrm{ZnO}: \mathrm{Zr}: \mathrm{F}$ thin solid films.

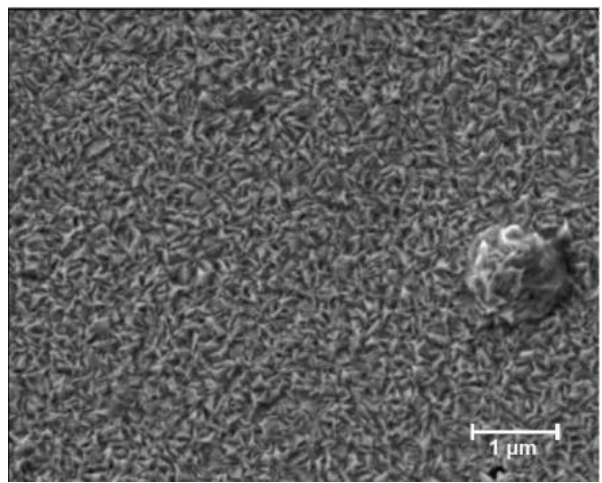

Figure 4: SEM micrograph of the studied $\mathrm{ZnO} \mathrm{Zr}$ :F thin film.

films by taking into account X-ray measurements. The atomic concentration of the elements present in the films could be expected to be very low, but unfortunately, its identification has not been carried out yet.

From Figure 4, it can be observed a representative panoramic view of one region of the samples obtained by Scanning Electronic Microscopy (SEM). The image shows evidence of structured morphology that can be obtained with 


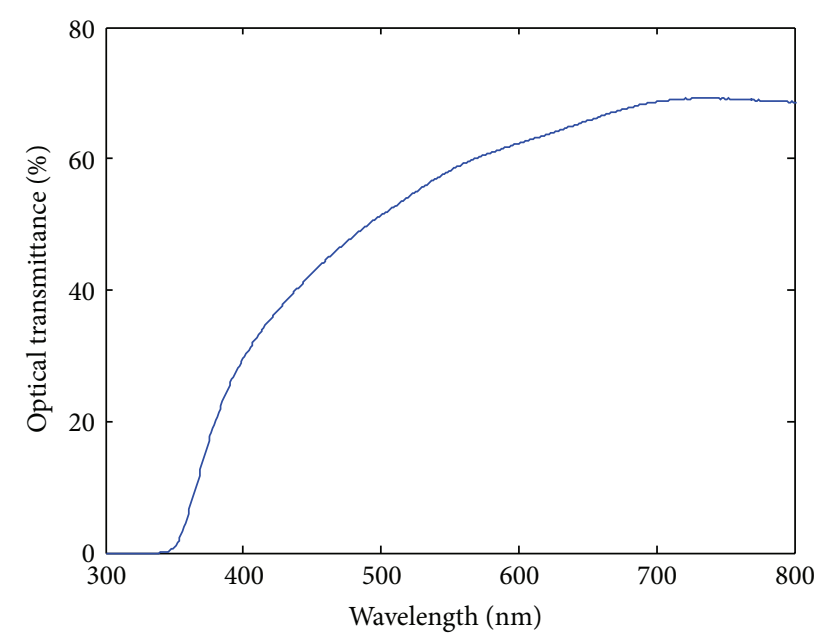

FIgURe 5: Optical transmittance spectrum of $\mathrm{ZnO} \mathrm{Zr}$ :F thin films.

the UST. The selected film thickness was in the order of $500 \mathrm{~nm}$, and the crystallite size of the films was about $35 \mathrm{~nm}$.

Figure 5 depicts the optical transmission spectrum of the studied sample. An absorbing edge towards the UV that starts at $350 \mathrm{~nm}$ can be clearly observed and associated to the substrate absorption. All the films were uniform and transparent in naked eyes. Optical transmittance remained high in the visible spectral range. Though the optical transparency of the film varied a bit with deposition conditions in the ultraviolet spectral range (which is irrelevant for the fabrication of TCO electrode applications), the position of their band edge did not vary significantly.

A high intensity optical beam at $532 \mathrm{~nm}$ wavelength was selected to observe if any involvement of multiphotonic interaction could be detected during the propagation of a nonresonant optical beam. A two-wave mixing system was previously calibrated using carbon disulfide $\left(\mathrm{CS}_{2}\right)$ with a thickness of $D=1 \mathrm{~mm}$, as a nonlinear medium with a well known third-order nonlinear susceptibility of $\left|\chi^{(3)}\right|=1.9 \times$ $10^{-12}$ esu [12]. A clear typical behavior of a two-photon absorption (TPA) effect was observed in the resulting films. The representative transmittance of the probe beam in the two-wave interaction for different angles, $\phi$, between the planes of polarization of the two incident beams is shown in Figure 6. The fit of the data was completed by using the expressions for describing the amplitudes of the transmitted fields derived elsewhere [13] as follows:

$$
\begin{array}{r}
E_{1 \pm}(z)=\left[E_{1 \pm}^{0} J_{0}\left(\Psi_{ \pm}^{(1)}\right)+\left(i E_{2 \pm}^{0}-i E_{3 \pm}^{0}\right) J_{1}\left(\Psi_{ \pm}^{(1)}\right)\right. \\
\left.-E_{4 \pm}^{0} J_{2}\left(\Psi_{ \pm}^{(1)}\right)\right] \exp \left(i \Psi_{ \pm}^{(0)}-\frac{\alpha(I) z}{2}\right), \\
E_{2 \pm}(z)=\left[E_{2 \pm}^{0} J_{0}\left(\Psi_{ \pm}^{(1)}\right)+\left(i E_{4 \pm}^{0}-i E_{1 \pm}^{0}\right) J_{1}\left(\Psi_{ \pm}^{(1)}\right)\right. \\
\left.-E_{3 \pm}^{0} J_{2}\left(\Psi_{ \pm}^{(1)}\right)\right] \exp \left(i \Psi_{ \pm}^{(0)}-\frac{\alpha(I) z}{2}\right),
\end{array}
$$

where $E_{1 \pm}(z)$ and $E_{2 \pm}(z)$ are the complex amplitudes of the circular components of the transmitted waves beams;

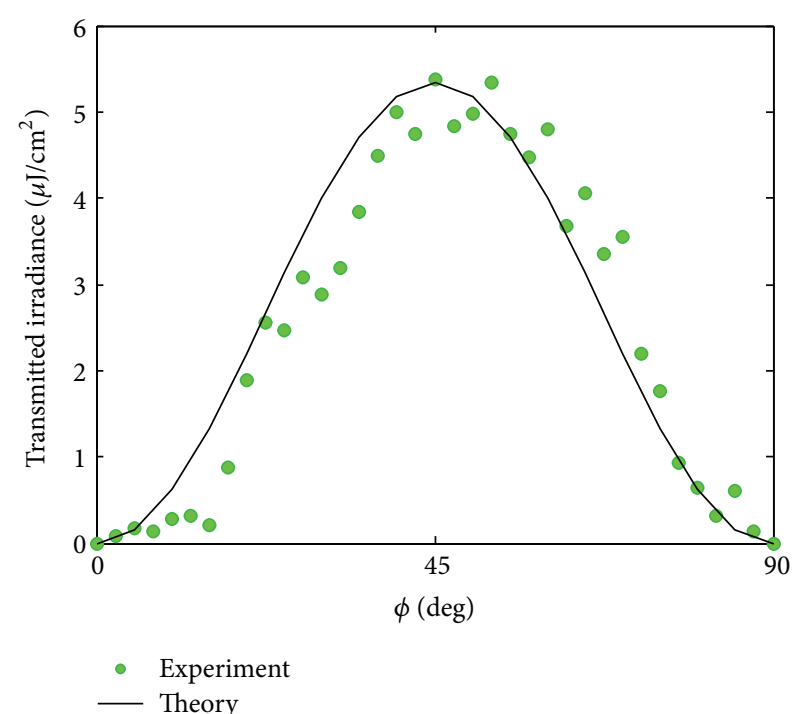

FIGURE 6: Transmitted irradiance of the probe beam versus angle $\phi$ between planes of polarization of the incident waves.

$E_{3 \pm}(z)$ and $E_{4 \pm}(z)$ are the amplitudes of the self-diffracted waves, while $E_{1 \pm}^{0}, E_{2 \pm}^{0}, E_{3 \pm}^{0}$, and $E_{4 \pm}^{0}$ are the amplitudes of the incident and self-diffracted waves at the surface of the sample; $\alpha(I)=\alpha_{o}+\beta I$ is the irradiance dependent absorption coefficient, where $\alpha_{o}$ and $\beta$ are the linear and two-photon absorption coefficients, respectively; $I$ is the total irradiance of the incident beams; $J_{m}\left(\Psi_{ \pm}^{(1)}\right)$ stands for the Bessel function of order $m, z$ is the thickness of the nonlinear media, and

$$
\begin{aligned}
\Psi_{ \pm}^{(0)}= & \frac{4 \pi^{2} z}{n_{0} \lambda} \\
\times & {\left[\left(A+\frac{n_{0} \beta}{2 \pi}\right) \sum_{j=1}^{4}\left|E_{j \pm}\right|^{2}\right.} \\
& \left.+\left(A+B+\frac{n_{0} \beta}{2 \pi}\right) \sum_{j=1}^{4}\left|E_{j \pm}\right|^{2}\right], \\
\Psi_{ \pm}^{(1)}=\frac{4 \pi^{2} z}{n_{0} \lambda} & \quad\left[\left(A+\frac{n_{0} \beta}{2 \pi}\right) \sum_{j=1}^{3} \sum_{k=2}^{4} E_{j \pm} E_{k \pm}^{*}\right. \\
& \left.\quad+\left(A+B+\frac{n_{0} \beta}{2 \pi}\right) \sum_{j=1}^{3} \sum_{k=2}^{4} E_{j \pm} E_{k \pm}^{*}\right],
\end{aligned}
$$

are the nonlinear phase changes. The optical wavelength is represented by $\lambda$, and the refractive index is $n_{0}$. The best fitting for the linear and nonlinear absorptive coefficients results in $\alpha_{0}=3.3 \times 10^{5} \mathrm{~m}^{-1}$ and $\beta=5.3 \times 10^{-8} \mathrm{~m} / \mathrm{W}$. 
TABLE 1: Photoconductive multiplexed results.

\begin{tabular}{lcccc}
\hline $\begin{array}{l}\text { Laser input 1 } \\
{\left[\mathrm{MW} / \mathrm{cm}^{2}\right]}\end{array}$ & $\begin{array}{c}\text { Laser input 2 } \\
{\left[\mathrm{MW} / \mathrm{cm}^{2}\right]}\end{array}$ & $\begin{array}{c}\text { Laser input 3 } \\
{\left[\mathrm{MW} / \mathrm{cm}^{2}\right]}\end{array}$ & $\begin{array}{c}\text { Photoconductive value at the output of the multiplexing system } \\
{[\mathrm{mho} / \mathrm{cm}]}\end{array}$ \\
\hline 280 & 0 & 0 & Parallel circuit & Series circuit \\
0 & 280 & 0 & 99.6 & 3.95 \\
280 & 280 & 0 & 174.3 & 3.95 \\
0 & 0 & 280 & 99.6 & 3.92 \\
280 & 0 & 280 & 174.3 & 6.95 \\
0 & 280 & 280 & 174.3 & 6.92 \\
280 & 280 & 280 & 249 & 27.66 \\
\hline
\end{tabular}

The error bar in the experimental data is around $\pm 5 \%$. It has been previously reported that $\mathrm{F}$ doping strongly contributes to the development of a third-order nonlinear optical response of $\mathrm{ZnO}$ [9]. Moreover, in this work, it has been observed that stronger optical Kerr nonlinearities can be obtained by $\mathrm{ZnO}: \mathrm{Zr}: \mathrm{F}$ films with the advantage of an improved conductivity. In comparison with a previous studied ZnO:F sample [9], this TPA effect seems to present a higher level in about one order of magnitude after the incorporation of Zr dopants. Apparently, this situation ought to be related to a dynamic modification in the transitions related to fluorine and zirconium impurities in the $\mathrm{ZnO}$ film. Furthermore, it was evaluated the nonlinear optical response of a pure $\mathrm{ZnO}$ film and it was not detected any important nonlinear third order signal; besides, the conductive properties for different processing routes in similar samples have been previously reported [11]. Further studies on the deposition of $\mathrm{ZnO}: \mathrm{Zr}: \mathrm{F}$ films and optimization of the implemented UST parameters should be performed in order to improve the film quality, crystallinity, and the nonlinear optical response of the samples with the deposition temperature and dopants concentration.

A photoconductive phenomenon was measured in the samples excited by the incidence of the nanosecond beam with $80 \mathrm{~mJ}$ of pulse energy and $6 \mathrm{~mm}$ diameter. The conductivity of the sample was about one order of magnitude higher than the stable measurement of about $8.3 \mathrm{mho} / \mathrm{cm} \pm 5 \%$ in darkness. An important inhibition of the photoconductive response was measured in the samples prepared with short aging times in the precursor solution. Thus, we assumed that the TPA effect could be responsible for the photoconductive phenomena in the sample, as it has been previously suggested for some other semiconductor materials $[14,15]$. The transmittance $T$ versus incident irradiance of a single beam interaction is shown in Figure 7. A good agreement in the fitting of the data confirms the presence of a TPA process into the sample. This resulting $\beta$ value is considerably lower than the same parameter previously reported for a $445 \mathrm{~nm}$ wavelength in a pure $\mathrm{ZnO}$ thin film originating a near-exciton resonance [16].

The measured conditions for the proposed photoconductive multiplexor configurations shown in Figure 2 are expressed in Table 1 . The error bar level is about $\pm 5 \%$.

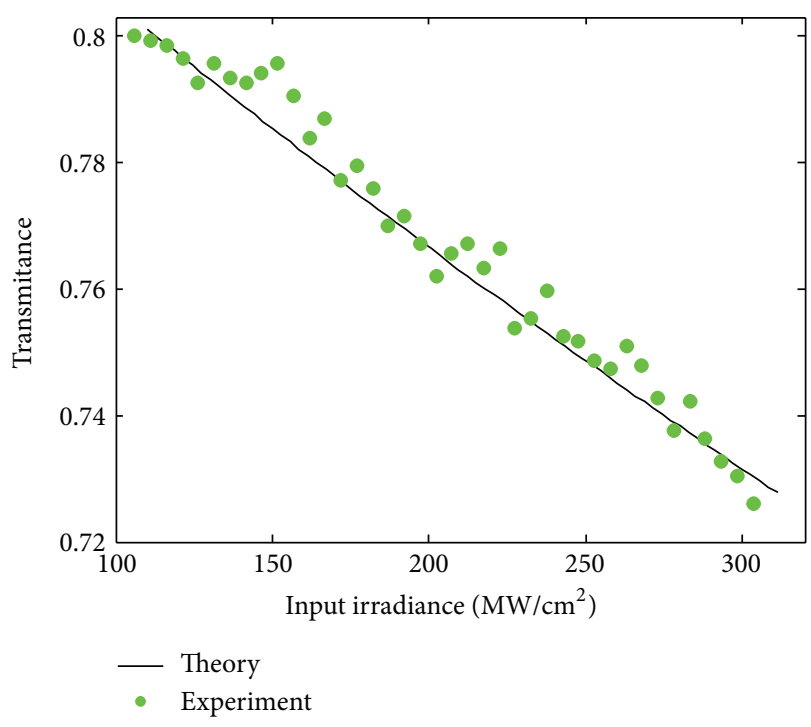

FIGURE 7: Nonlinear optical transmittance of a single beam in the studied samples.

It is clear that the photoconductive response seems to be different but similar in the two studied circuits; however, regarding the important absorptive nonlinearities exhibited by the samples, the use of a nonlinear behavior can be considered for adjusting the multiplexing performance. The ultrafast optical and excitonic responses of $\mathrm{ZnO}$ media have attracted the attention of many studies in order to propose different device applications $[16,17]$. It has been demonstrated that strong and ultrafast nonlinear optical properties can be exhibited by TCO thin solid films manufactured on $\mathrm{ZnO}$ media. All-optical switching, optical Kerr modulators, and photoconductive systems based on nonlinear optical absorption have been also proposed [18]. Several motivations for the study of $\mathrm{ZnO}$ films have been associated to their conductive response; nevertheless in this work; we highlight the possibility for finding additional non-resonant photoconductive applications that can be also stimulated in combination with their optical limiting performance in transparent platforms based on $\mathrm{ZnO}$ doped films. 


\section{Conclusions}

Within this work, $\mathrm{ZnO}: \mathrm{Zr}$ :F thin solid films with photoconductive and nonlinear optical properties were presented. A photoconductive multiplexing system based on the samples was proposed. In regard to the optical and electrical measurements presented, it is estimated that the films can be highly attractive for transparent platforms with potential application for developing photoconductive nonlinear optical devices.

\section{Acknowledgment}

The authors gratefully acknowledge the financial support from the Instituto Politécnico Nacional, Consejo Nacional de Ciencia y Tecnología.

\section{References}

[1] P. S. Patil, "Versatility of chemical spray pyrolysis technique," Materials Chemistry and Physics, vol. 59, no. 3, pp. 185-198, 1999.

[2] M. Krunks and E. Mellikov, "Zinc oxide thin films by the spray pyrolysis method," Thin Solid Films, vol. 270, no. 1-2, pp. 33-36, 1995.

[3] B. Mukherjee, B. Varghese, M. Zheng et al., "Synthesis, characterization and electrical properties of hybrid $\mathrm{Zn}_{2} \mathrm{GeO}_{4}-\mathrm{ZnO}$ beaded nanowire arrays," Journal of Crystal Growth, vol. 346, no. 1, pp. 32-39, 2012.

[4] R. García-Gutiérrez, M. Barboza-Flores, D. Berman-Mendoza, R. Rangel-Segura, and O. E. Contreras-López, "Luminescence and structure of $\mathrm{ZnO}$ grown by physical vapor deposition," Advances in Materials Science and Engineering, vol. 2012, Article ID 872597, 5 pages, 2012.

[5] J. G. Lu, T. Kawaharamura, H. Nishinaka, Y. Kamada, T. Ohshima, and S. Fujita, "Zno-based thin films synthesized by atmospheric pressure mist chemical vapor deposition," Journal of Crystal Growth, vol. 299, no. 1, pp. 1-10, 2007.

[6] A. Tiburcio-Silver, A. Sanchez-Juarez, and A. Avila-Garcia, "Properties of gallium-doped $\mathrm{ZnO}$ deposited onto glass by spray pyrolysis," Solar Energy Materials and Solar Cells, vol. 55, no. 1-2, pp. 3-10, 1998.

[7] E. Gungor and T. Gungor, "Effect of the substrate movement on the optical properties of $\mathrm{ZnO}$ thin films deposited by ultrasonic spray pyrolysis," Advances in Materials Science and Engineering, vol. 2012, Article ID 594971, 7 pages, 2012.

[8] G. K. Paul, S. Bandyopadhyay, S. K. Sen, and S. Sen, "Structural, optical and electrical studies on sol-gel deposited $\mathrm{Zr}$ doped $\mathrm{ZnO}$ films," Materials Chemistry and Physics, vol. 79, no. 1, pp. 71-75, 2003.

[9] C. Torres-Torres, J. H. Castro-Chacón, L. Castañeda et al., "Ultrafast nonlinear optical response of photoconductive $\mathrm{ZnO}$ films with fluorine nanoparticles," Optics Express, vol. 19, no. 17, pp. 16346-16355, 2011.

[10] A. Guillén-Santiago, M. D. L. L. Olvera, A. Maldonado, R. Asomoza, and D. R. Acosta, "Electrical, structural and morphological properties of chemically sprayed F-doped $\mathrm{ZnO}$ films: effect of the ageing-time of the starting solution, solvent and substrate temperature," Physica Status Solidi A, vol. 201, no. 5, pp. 952-959, 2004.

[11] A. Maldonado, S. Tirado-Guerra, and M. de la L. Olvera, "Chemically sprayed $\mathrm{ZnO}$ :(F, $\mathrm{Zr}$ ) thin films: effect of starting solution ageing time and substrate temperature on the physical properties," Journal of Physics and Chemistry of Solids, vol. 70, no. 3-4, pp. 571-575, 2009.

[12] R. W. Boyd, Nonlinear Optics, Academic Press, San Diego, Calif, USA, 1992.

[13] C. Torres-Torres, L. Castaneda, M. Trejo-Valdez, A. Maldonado, and R. Torres-Martinez, "Participation of the third order optical nonlinearities in nanostructured silver doped zinc oxide thin solid films," Journal of Nanomaterials, vol. 2012, Article ID 353061, 5 pages, 2012.

[14] Y.-P. Chan, J.-H. Lin, C.-C. Hsu, and W.-F. Hsieh, "Nearresonant high order nonlinear absorption of $\mathrm{ZnO}$ thin films," Optics Express, vol. 16, no. 24, pp. 19900-19908, 2008.

[15] H. Folliot, M. Lynch, A. L. Bradley et al., “Two-photon-induced photoconductivity enhancement in semiconductor microcavities: a theoretical investigation," Journal of the Optical Society of America B, vol. 19, no. 10, pp. 2396-2402, 2002.

[16] T. Shih, E. Mazur, J.-P. Richters, J. Gutowski, and T. Voss, "Ultrafast exciton dynamics in $\mathrm{ZnO}$ : excitonic versus electronhole plasma lasing," Journal of Applied Physics, vol. 109, no. 4, Article ID 043504, 2011.

[17] M. Li, G. Xing, G. Xing et al., "Origin of green emission and charge trapping dynamics in $\mathrm{ZnO}$ nanowires," Physical Review $B$, vol. 87, no. 11, Article ID 115309, 8 pages, 2013.

[18] C. Torres-Torres, M. L. García-Cruz, L. Castañeda et al., "Photoconductivity, photoluminescence and optical Kerr nonlinear effects in zinc oxide films containing chromium nanoclusters," Journal of Luminescence, vol. 132, no. 4, pp. 1083-1088, 2012. 

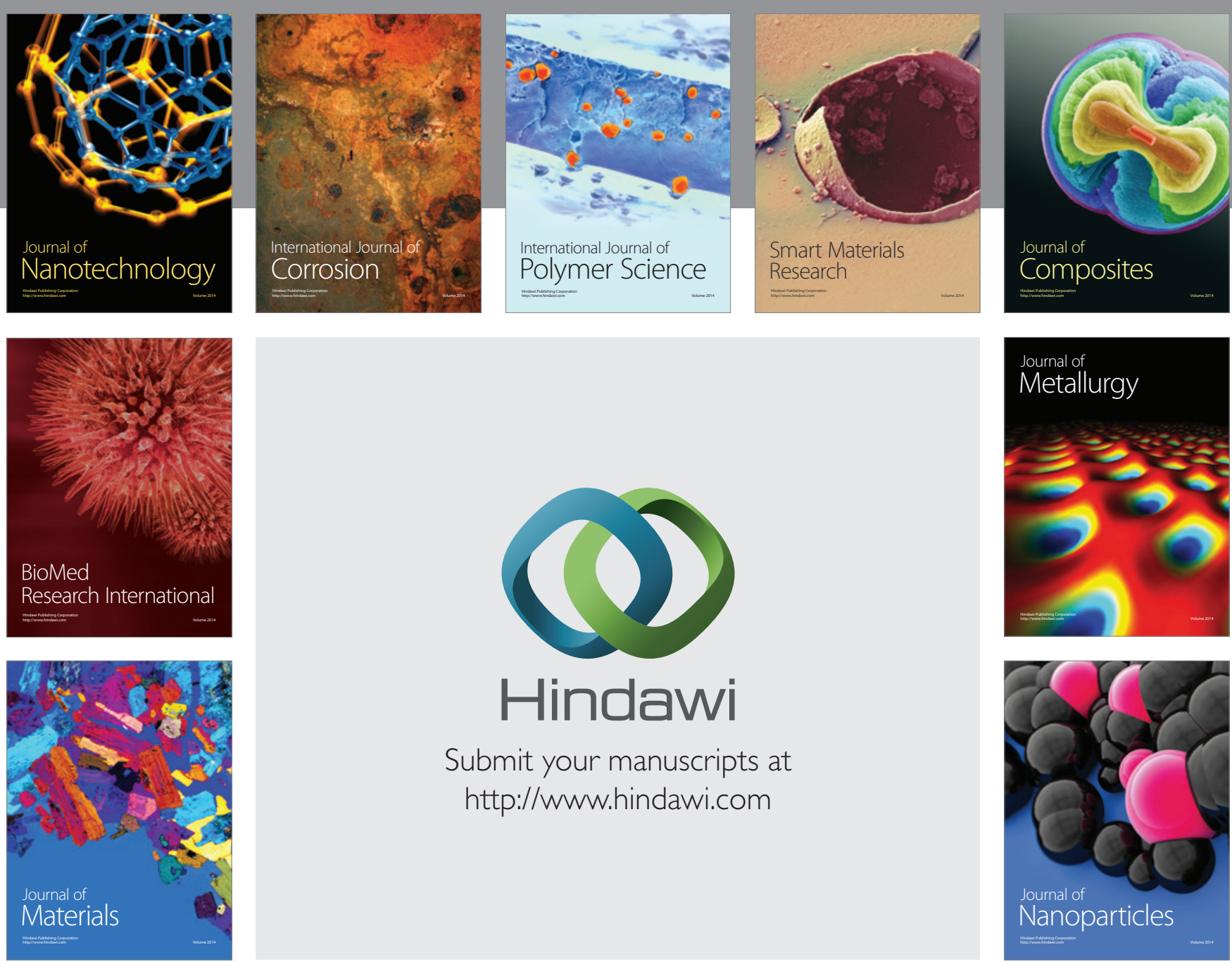

Submit your manuscripts at http://www.hindawi.com
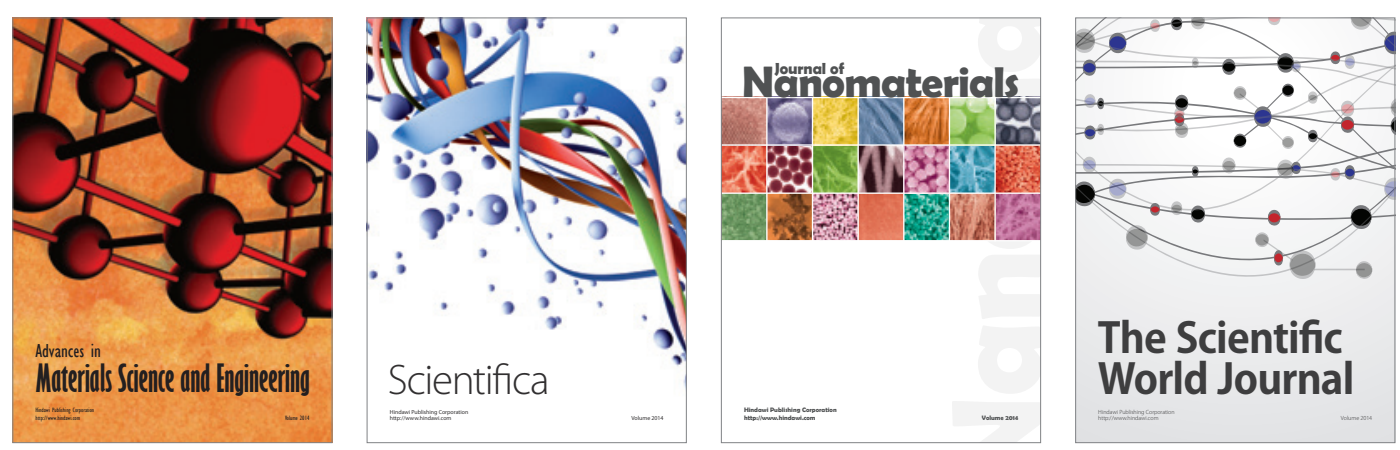

\section{The Scientific World Journal}
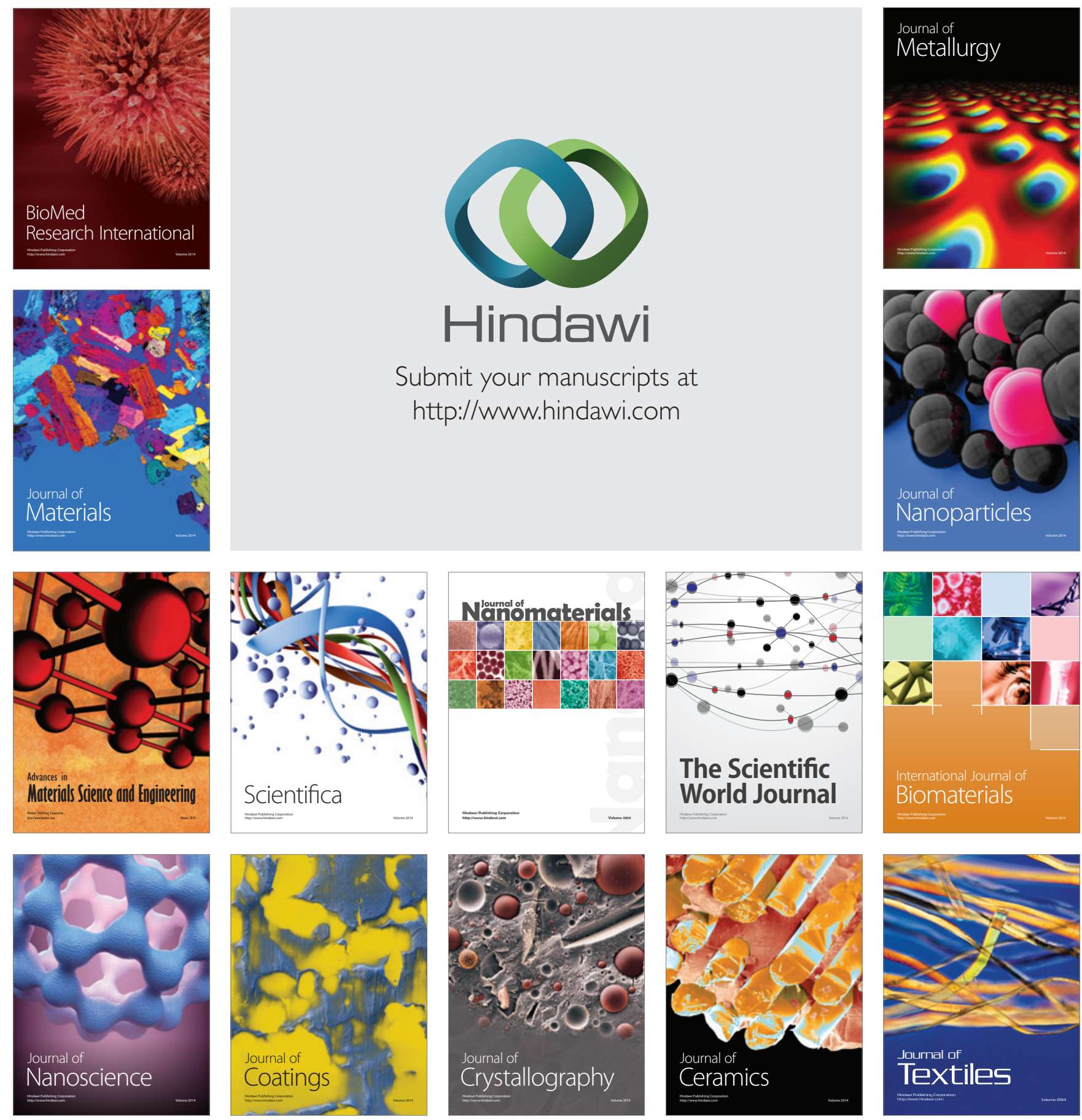